\section{OXIDATIVE STRESS ORIGINATING IN THE MITOCHONDRIA DAMAGES TELOMERES SUFFICIENT TO DRIVE CERTAIN FEATURES OF T CELL DYSFUNCTION}

${ }^{1}$ Dayana Rivadeneira*, ${ }^{1}$ Jess Yana, ${ }^{1}$ Sanjana Thosar, ${ }^{2}$ Marcel Bruchez, ${ }^{1}$ Patricia Lynn, ${ }^{1}$ Greg Delgoffe. ' University of Pittsburgh, Pittsburgh, PA, United States; ${ }^{2}$ Carnegie Mellon University, Pittsburgh, PA, United States

Background The functional state of infiltrating lymphocytes is a critical determinant of antitumor immunity and immunotherapy response. Key factors responsible for driving $\mathrm{T}$ cell dysfunction are metabolic barriers such as nutrient competition, low oxygen tension and damaging byproducts, like reactive oxygen species (ROS). ROS are critical contributors to $\mathrm{T}$ cell dysfunction observed during aging as well as in the tumor microenvironment. While we have shown ROS accumulation drives $\mathrm{T}$ cell exhaustion in part by altering signaling, ROS can affect other cellular functions further contributing to $\mathrm{T}$ cell dysfunction. One of the main downstream consequences observed with ROS accumulation is DNA damage, in particular telomeric DNA. However, little is known on whether telomeric shortening vs damage and the associated response affects $\mathrm{T}$ cell fate and function.

Methods We performed telomeric analyses of endogenous tumor-infiltrating $\mathrm{T}$ cells. In vitro, direct induction of oxidative stress in mitochondria or telomeres was performed using a photosensitizer approach employing fluorogen-activating peptide (FAP) ${ }^{1}$ targeted to the mitochondria (COXVIII-FAP) or to the telomeric shelterin protein TRF1, which produces singlet oxygen and 8-oxoguanine specifically in the mitochondria or at the telomeres. ${ }^{2}$ Our lab generated mouse models to express this telomeric-FAP (Rosa26-LSL-TRF1-FAP) or mitochondrialFAP (Rosa26-LSL-COXVIII-FAP) specifically in $\mathrm{T}$ cells (Cd4Cre).

Results Telomere analysis of tumor-infiltrating exhausted (PD1hiTim3+) vs non-exhausted (PD1int) cells revealed exhausted T cells do not have shorter telomeres (like senescent $\mathrm{T}$ cells), but rather damaged telomeres. Using a photosensitizer strategy to specifically induce oxidative damage to the mitochondria, we recapitulated our previous work in vitro, resulting in ROS cascades, severe T cell dysfunction, and damage to telomeres. We next directly assessed the role of telomere oxidative damage using a telomeric-targeted photosensitizer, showing oxidative damage to telomeres promotes a persistent $\mathrm{T}$ cell dysfunction, resulting in sustained Tim3 and PD1 expression and severely decreased functions. However, oxidative damage to telomeric DNA alone does not induce all of the features of $\mathrm{T}$ cell exhaustion, suggesting that telomere damage is a crucial piece, but not fully sufficient to drive $\mathrm{T}$ cell dysfunction.

Conclusions Our data support a model where oxidative stress originating in the mitochondria alters cellular biology in part by damaging telomeric DNA. While this activity does not shorten telomeres, it induces stress responses sufficient to deviate differentiation into a dysfunctional phenotype. While telomeric damage alone does not fully recapitulate exhaustion, it nevertheless remains a crucial part of the dysfunctional phenotype in tumor infiltrating lymphocytes.

\section{REFERENCES}

1. He J, Wang $Y$, Missinato MA, Onuoha E, Perkins LA, Watkins SC, St Croix CM, Tsang M, Bruchez MP. A genetically targetable near-infrared photosensitizer Nat Methods 2016;13:263-268.

2. Fouquerel E, Barnes RP, Uttam S, Watkins SC, Bruchez MP, Opresko PL. Targeted and persistent 8-Oxoguanine base damage at telomeres promotes telomere loss and crisis. Mol Cell 2019;75(1):117-130.e6. 